\title{
National Emphysema Treatment Trial redux: Accentuating the positive
}

\author{
Pablo Gerardo Sanchez, MD, John Charles Kucharczuk, MD, Stacey Su, MD, Larry Robert Kaiser, MD, and \\ Joel David Cooper, MD
}

\begin{abstract}
Objective: Under the Freedom of Information Act, we obtained the follow-up data of the National Emphysema Treatment Trial (NETT) to determine the long-term outcome for "a heterogeneous distribution of emphysema with upper lobe predominance," postulated by the NETT hypothesis to be optimal candidates for lung volume reduction surgery.
\end{abstract}

\begin{abstract}
Methods: Using the NETT database, we identified patients with heterogeneous distribution of emphysema with upper lobe predominance and analyzed for the first time follow-up data for those receiving lung volume reduction surgery and those receiving medical management. Furthermore, we compared the results of the NETT reduction surgery group with a previously reported consecutive case series of 250 patients undergoing bilateral lung volume reduction surgery using similar selection criteria.
\end{abstract}

Results: Of the 1218 patients enrolled, 511 (42\%) conformed to the NETT hypothesis selection criteria and received the randomly assigned surgical or medical treatment (surgical $=261$; medical $=250$ ). Lung volume reduction surgery resulted in a 5-year survival benefit $(70 \%$ vs $60 \% ; P=.02)$. Results at 3 years compared with baseline data favored surgical reduction in terms of residual volume reduction $(25 \%$ vs $2 \% ; P<.001)$, University of California San Diego dyspnea score (16 vs 0 points; $P<.001$ ), and improved St George Respiratory Questionnaire quality of life score (12 points vs 0 points; $P<.001$ ). For the 513 patients with a homogeneous pattern of emphysema randomized to surgical or medical treatment, lung volume reduction surgery produced no survival advantage and very limited functional benefit.

Conclusions: Patients most likely to benefit from lung volume reduction surgery have heterogeneously distributed emphysema involving the upper lung zones predominantly. Such patients in the NETT trial had results nearly identical to those previously reported in a nonrandomized series of similar patients undergoing lung volume reduction surgery. (J Thorac Cardiovasc Surg 2010;140:564-72)

Lung volume reduction surgery (LVRS) is a palliative procedure for selected patients with severe emphysema whose respiratory mechanics are severely impaired owing to hyperinflation of the lungs and thorax. The goal of LVRS is the reduction of hyperinflation by removal of the most destroyed portions of the lung, thus improving respiratory mechanics, dyspnea, exercise tolerance, and quality of life. The concept of lung volume reduction was introduced by Brantigan, Mueller, and Kress ${ }^{1}$ in 1959. Their procedure resulted in a high operative mortality, and although many of their patients reported clinical improvement, the lack of objective documentation led to skepticism as to the value of the procedure.

\footnotetext{
From the Division of Thoracic Surgery, Department of Surgery, University of Pennsylvania, Philadelphia, Pa.

Supported in part by a grant from the Lavin Family Foundation.

Disclosures: None.

Read at the Eighty-ninth Annual Meeting of The American Association for Thoracic Surgery, Boston, Massachusetts, May 9-13, 2009.

Received for publication June 30, 2009; revisions received March 8, 2010; accepted for publication March 16, 2010.

Address for reprints: Joel D. Cooper, MD, Division of Thoracic Surgery, University of Pennsylvania, 3400 Spruce St, Silverstein 6th, Philadelphia, PA 19104 (E-mail: Joel.cooper@uphs.upenn.edu).

0022-5223/\$36.00

Copyright (c) 2010 by The American Association for Thoracic Surgery doi:10.1016/j.jtcvs.2010.03.050
}

LVRS was reintroduced in $1995 .^{2}$ Results from 250 consecutive patients undergoing bilateral LVRS were subsequently reported ${ }^{3}$ and demonstrated significant objective and subjective benefit. Other reports followed and confirmed the benefit of LVRS. ${ }^{4-9}$

The reintroduction of LVRS and the documented functional benefit achieved ${ }^{2}$ attracted a great deal of interest in the medical and surgical community. However, the rapid dissemination of the LVRS procedure raised serious questions regarding its value and its appropriate application. Patient selection, surgical approach, and technique were variable as were morbidity, mortality, and reported outcomes. Data analyzed from Medicare claims with the LVRS billing codes showed a high mortality rate and significant postoperative, short-term, and long term hospitalizations for these patients. ${ }^{4}$ For these reasons the Health Care Finance Administration suspended payment for the procedure and, together with the National Heart, Lung, and Blood Institute and the Agency for Healthcare Policy and Research, conducted a prospective randomized clinical trial of bilateral LVRS known as the National Emphysema Treatment Trial (NETT). The rationale and design of the NETT have been reported in detail. ${ }^{10}$ Screening of prospective patients began in October 1997, and randomization 


\section{Abbreviations and Acronyms}

$\mathrm{FEV}_{1}=$ forced expiratory volume in 1 second

LVRS $=$ lung volume reduction surgery

NETT $=$ National Emphysema Treatment Trial

$\mathrm{RV}=$ residual volume

between LVRS and medical management began in January 1998.

In 2001 the NETT research group published a preliminary report identifying a subgroup of NETT patients at high risk of death after LVRS, ${ }^{11}$ and such patients were no longer considered eligible for the trial. Patient entry for the trial ended in July 2002, at which time a total of 1218 patients had been randomly assigned to undergo either LVRS or medical management. The initial NETT report on the early outcome of the overall study was published in May 2003. ${ }^{12}$ Long-term follow-up of the NETT trial, with a median follow-up of 4.3 years, was reported in $2006 .^{13}$

Under the Freedom of Information Act, we have obtained and analyzed the NETT data set released as of May 2006. Using those data, we have specifically analyzed for the first time the outcome of the medical and surgical patients who met the original NETT study hypothesis criteria (which we refer to as the target group), as well as other subgroups who did not. We further compared the results of these patients from the NETT study with results we previously reported in 250 consecutive patients whose selection criteria closely matched those of the patients who met the NETT hypothesis.

\section{METHODS}

After institutional review board approval and signature of a data distribution agreement between the University of Pennsylvania and the National Heart, Lung, and Blood Institute, we received an electronic data set entitled "Limited Access Database Documentation" containing patient data up to May 2006. This extensive database consisted of 286 pages documenting the data fields collected for each patient under the NETT protocol. This electronic data set was then analyzed with the SPSS 17.0 statistical software package (SPSS, Inc, Chicago, Ill).

A total of 1218 patients were enrolled in the NETT trial. After a 6- to 10week period of pulmonary rehabilitation, patients were then randomized to either medical therapy or medical therapy plus bilateral LVRS. The median time from randomization to surgery was 10 days, with $88 \%$ of patients assigned to LVRS undergoing the procedure within 14 days.

During the development of the NETT protocol, an analysis of available LVRS data was undertaken, much provided by the NETT centers, to help guide the study protocols. This generated a hypothesis by the NETT steering committee as follows: "Patients who improve (after LVRS) will have preoperative (A) significant loss of lung elastic recoil (ie, emphysema) and (B) heterogeneously distributed emphysema involving the upper lung zones predominantly." 14

The ultimate inclusion criteria for the NETT trial, however, were not limited to only such patients. The final selection criteria were "formulated to include patients with a diverse distribution of emphysema to examine the effect of the anatomic distribution of disease on the response to therapy.",10

We identified the subgroup of patients who met the NETT hypothesis in the following manner. In the NETT trial, the overall pattern of craniocaudal distribution of emphysema (predominance criteria) was determined by a radiologist who had been trained in the study protocol. On the basis of a chest computed tomographic scan, the radiologist recorded the pattern of emphysema as belonging to 1 of the following unique categories:

- Upper lobe predominant

- Lower lobe predominant

- Superior segments of lower lobes predominantly

- Diffuse

We used this data field to identify patients who had upper lobe predominant disease.

To identify which patients met the definition of a heterogeneous pattern of emphysema, the NETT radiologist divided each lung into 3 apicalto-basal zones, and the severity of emphysema in each zone was scored visually on a scale of 0 to 4 with a grade of 0 indicating no emphysema and a grade of 4 indicating the presence of emphysema in more than $75 \%$ of the lung zone. Heterogeneous emphysema was defined by the NETT as a difference in the severity score of at least 2 integers between any 2 zones in at least 1 lung. Otherwise, the distribution of emphysema was classified as diffuse (homogenous).

Patients who met the NETT hypothesis were those who met both the NETT criteria for heterogeneously distributed emphysema and the NETT criteria for upper lobe predominant disease. It should be noted that under the NETT definitions, a patient could be classified as both upper lobe predominant and homogeneous if he or she met the criteria for upper lobe predominance, but if the severity of the upper lobe emphysema on either side was not scored greater than 1 integer higher than the other 2 lung zones on the basis of the severity scoring.

\section{Statistical Analysis}

All statistical analysis of NETT patients was based on patients who received the assigned treatment. Descriptive statistics are expressed as mean \pm SD unless otherwise specified. Categorical data are expressed as counts and proportions. Comparisons were done with paired, 2-tailed $t$ tests for means of normally distributed continuous variables and Wilcoxon rank sum tests for skewed data. Either $\chi^{2}$ or Fisher's exact tests were used to analyze differences among the categorical data. Kaplan-Meier estimation was used to predict survival.

\section{RESULTS}

Between January 1998 and July 2002, 1218 patients were randomized in the NETT trial. Of the 608 patients assigned to the surgical group, 571 received bilateral LVRS. Of the 610 patients assigned to medical therapy, 566 patients received the assigned therapy. Applying the NETT criteria for the radiographic pattern and distribution of emphysema, 261 of the patients receiving bilateral LVRS corresponded to the target group (heterogeneous upper lobe predominant disease) whereas 257 had diffuse disease and 53 had a pattern of heterogeneous lower lobe predominance. For the medical group, 250 patients met the target group criteria whereas 256 had diffuse disease and 60 had a pattern of heterogeneous lower lobe predominance.

\section{Patients With Heterogeneous Upper Lobe Predominance}

For the 261 LVRS patients in this group the mean age was 65.3 years, and for the medical group the mean age was 66.5 years. The median follow-up was 4.3 years. 

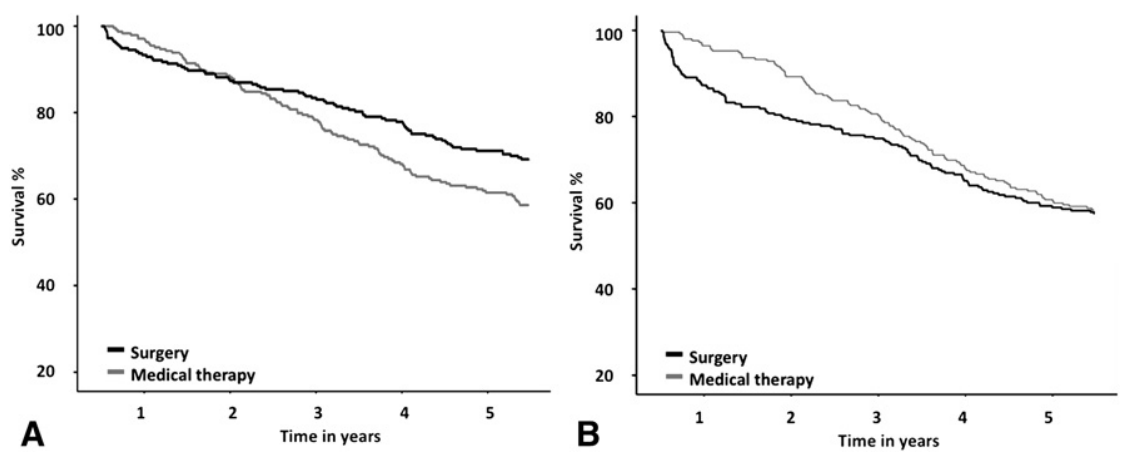

FIGURE 1. Kaplan-Meier survival curves on the treated patients. A, Heterogeneous upper lobe predominant group: LVRS versus medical management, $P=.02$. B, Homogeneous NETT LVRS versus medical management, $P=.49$.

Ninety-day mortality from the date of randomization was $13(5 \%)$ patients for the surgical group and $4(1.6 \%)$ patients for the medical group. Figure 1 shows the overall survival for the 2 groups. Survival at 3 years was $81 \%$ for the surgical group and $74 \%$ for the medical group $(P=.05)$. At 5 years survival was $70 \%$ for the surgical group and $60 \%$ for the medical group $(P=.02)$.

The 2 primary outcome measures chosen by the NETT trial were survival and maximum exercise capacity measured in terms of watts. Secondary measures were quality of life and related disease-specific symptoms. Other out- come measures were pulmonary function and gas exchange, oxygen requirement, and 6-minute walk distance.

Table 1 shows selected pulmonary function studies, exercise tolerance, and quality of life assessment for the target group patients in the surgical and medical groups. The NETT trial achieved excellent balance between the 2 groups in terms of baseline parameters. At all postoperative time periods, the surgical group showed highly significant benefit compared with medical treatment in terms of forced expiratory volume in 1 second $\left(\mathrm{FEV}_{1}\right)$, residual volume $(\mathrm{RV})$, dyspnea score, and quality of life. The same was true for

TABLE 1. Surgery versus medical therapy: Bilateral LVRS in heterogeneous upper lobe predominant group

\begin{tabular}{|c|c|c|c|c|c|}
\hline & $\begin{array}{c}\text { Preop: } \\
\text { Surg }=261 \\
\text { MT }=250 \\
\end{array}$ & $\begin{array}{c}\text { Six months: } \\
\text { Surg }=224 \\
\text { MT }=183 \\
\end{array}$ & $\begin{array}{c}\text { One year: } \\
\text { Surg }=199 \\
\text { MT }=153 \\
\end{array}$ & $\begin{array}{c}\text { Two years: } \\
\text { Surg }=167 \\
\text { MT }=121 \\
\end{array}$ & $\begin{array}{c}\text { Three years: } \\
\text { Surg }=116 \\
\text { MT }=72\end{array}$ \\
\hline \multicolumn{6}{|c|}{$\mathrm{FEV}_{1}($ mean $\pm \mathrm{SD}[\mathrm{L}], \%$ predicted $)$} \\
\hline Surgery & $0.7 \pm 0.2(26 \%)$ & $1.0 \pm 0.4(37 \%) \ddagger$ & $1.0 \pm 0.3(36 \%) \ddagger$ & $1.0 \pm 0.3(36 \%) \ddagger$ & $0.9 \pm 0.3(33 \%)^{*}$ \\
\hline MT & $0.7 \pm 0.2(26 \%)$ & $0.7 \pm 0.2(26 \%)$ & $0.8 \pm 0.3(27 \%)$ & $0.8 \pm 0.3(27 \%)$ & $0.8 \pm 0.3(27 \%)$ \\
\hline \multicolumn{6}{|c|}{$\mathrm{RV}($ mean $\pm \mathrm{SD}(\mathrm{L}), \%$ predicted $)$} \\
\hline Surgery & $5.0 \pm 1.0(226 \%)$ & $3.4 \pm 1.0(157 \%) \ddagger$ & $3.6 \pm 1.0(163 \%) \ddagger$ & $3.6 \pm 1.0(160 \%) \ddagger$ & $3.7 \pm 1.0(163 \%) \ddagger$ \\
\hline MT & $5.0 \pm 1.0(227 \%)$ & $5.0 \pm 1.0(227 \%)$ & $5.0 \pm 1.0(227 \%)$ & $5.0 \pm 1.0(227 \%)$ & $4.9 \pm 1.0(211 \%)$ \\
\hline \multicolumn{6}{|c|}{$\mathrm{UCSD}($ mean $\pm \mathrm{SD})$} \\
\hline Surgery & $65 \pm 19$ & $41 \pm 23 \ddagger$ & $42 \pm 23 \ddagger$ & $46 \pm 24 \ddagger$ & $49 \pm 26 \ddagger$ \\
\hline MT & $66 \pm 19$ & $65 \pm 21$ & $66 \pm 20$ & $67 \pm 22$ & $66 \pm 22$ \\
\hline \multicolumn{6}{|c|}{ St George (mean \pm SD) } \\
\hline Surgery & $56 \pm 13$ & $39 \pm 16 \ddagger$ & $39 \pm 18 \ddagger$ & $42 \pm 17 \ddagger$ & $44 \pm 18 \ddagger$ \\
\hline MT & $57 \pm 13$ & $55 \pm 14$ & $57 \pm 14$ & $56 \pm 14$ & $57 \pm 15$ \\
\hline \multicolumn{6}{|c|}{ Workload (watts, mean \pm SD) } \\
\hline Surgery & $36 \pm 22$ & $48 \pm 22 \ddagger$ & $49 \pm 23 \ddagger$ & $49 \pm 24 \dagger$ & $43 \pm 23$ \\
\hline MT & $35 \pm 20$ & $37 \pm 23$ & $38 \pm 24$ & $40 \pm 24$ & $38 \pm 25$ \\
\hline \multicolumn{6}{|c|}{ Six-minute walk (feet, mean \pm SD) } \\
\hline Surgery & $1166 \pm 317$ & $1354 \pm 304 \ddagger$ & $1339 \pm 329 \ddagger$ & $1272 \pm 302 \ddagger$ & ฯ \\
\hline MT & $1120 \pm 298$ & $1165 \pm 311$ & $1128 \pm 343$ & $1136 \pm 350$ & ฯ \\
\hline
\end{tabular}


maximum workload with the exception of the 3-year data, at which time the difference between the LVRS group and the medical group did not achieve statistical significance but favored the LVRS group.

After LVRS, improvement in $\mathrm{FEV}_{1}$ over baseline values was $42 \%, 35 \%$, and $26 \%$ at 6 months, 1 year, and 3 years, respectively. The decrease in RV was $30 \%, 28 \%$, and $25 \%$ at the same time periods. For LVRS patients the workload improved by $34 \%, 33 \%$, and $18 \%$ at 6 months, 1 year, and 3 years, respectively.

Dyspnea and health-related quality of life as measured by the St George Respiratory Questionnaire both showed significant benefit for LVRS throughout the initial 3-year follow-up period.

\section{Bilateral LVRS for Upper Lobe Heterogeneous NETT Group Compared With Previous Consecutive Case Series}

Ciccone and associates ${ }^{3}$ previously reported results in 250 consecutive patients undergoing bilateral LVRS between January 1993 and June 2000. All patients had heterogeneously distributed emphysema, of whom $229(92 \%)$ had upper lobe predominance and the remaining $8 \%$ had lower lobe predominance. The mean age was $62 \pm 8$ years. Follow-up ranged from 1.8 to 9.1 years, with a median of 4.4 years. One patient was lost to follow-up. More than $95 \%$ of evaluable patients had objective pulmonary function data collected at each follow-up time point out to 5 years postoperatively.

Figure 2, A, depicts the Kaplan-Meier survival curve for the 261 NETT LVRS target group compared with the 250 patients in the Ciccone report. ${ }^{3}$ Table 2 shows results in terms of $\mathrm{FEV}_{1}, \mathrm{RV}$, and 6-minute walk test for these same patients. It will be noted that the baseline RV in the case series group was higher than in the NETT group $(5.8 \pm 1.3 \mathrm{~L}$ vs $5.0 \pm 1 \mathrm{~L})$. However, the baseline values for $\mathrm{FEV}_{1}$ and 6-minute walk test are virtually identical for the 2 groups. Figure $2, B$, depicts the post-LVRS change in the $\mathrm{FEV}_{1}$ in the 2 groups. The reduction in RV after LVRS at 6 months, 1 year, and 3 years was $31 \%$ versus $31 \%, 29 \%$ versus $27 \%$, and $28 \%$ versus $24 \%$ for the Ciccone series ${ }^{3}$ and the NETT series, respectively.

\section{LVRS Compared With Medical Treatment for NETT Patients With Homogeneous Emphysema Distribution}

We compared the results of bilateral LVRS versus medical therapy for the 518 NETT patients having a homogeneous pattern of emphysema. A total of 257 patients were randomized to LVRS and 261 patients to medical therapy. Figure 1 depicts the survival curve for the 2 groups. It is apparent that survival is similar for the 2 groups at 5 years. Table $3 \mathrm{com}-$ pares pulmonary function, dyspnea, quality of life, and maximal workload for the 2 groups. Although some measures show statistically significant benefit in favor of LVRS at 3 years, it is apparent that the magnitude of benefit is considerably diminished compared with the benefit of LVRS for patients with heterogeneous upper lobe predominant disease, shown in Table 1.

\section{Limitations of NETT Analysis}

The NETT survival data were complete out to 5 years, but there was significant lack of follow-up data for other parameters, which limited our analysis after year 3 of follow-up. We defined missing data at each time point as a lack of data for patients known to be alive at the time (1 year, 2 year, etc). For maximum workload, 1 of the 2 primary end points of the trial, and for pulmonary function studies, data were missing at 1 year, 2 years, and 3 years for $13 \%, 21 \%$, and $36 \%$ of the LVRS patients, respectively, and for $30 \%$, $35 \%$, and $43 \%$ of the medical patients, respectively.

\section{DISCUSSION}

The NETT trial represents the only randomized clinical trial of LVRS with a significant number of patients and well-defined outcome measures. Just as the LVRS procedure itself generated considerable controversy, so too did the methodology, selection process, and reporting of results of the NETT trial. Among the factors contributing to the controversy were changes in the selection criteria in the midst

TABLE 2. Comparisons Ciccone and associates ${ }^{3}$ and NETT data for LVRS

\begin{tabular}{|c|c|c|c|c|}
\hline & $\begin{array}{c}\text { Preop: Ciccone } \\
\text { et al: } 250 \text { NETT: } 261\end{array}$ & $\begin{array}{l}\text { Six months: Ciccone } \\
\text { et al: } 231 \text { NETT: } 224\end{array}$ & $\begin{array}{c}\text { One year: Ciccone } \\
\text { et al: } 225 \text { NETT: } 199\end{array}$ & $\begin{array}{l}\text { Three years: Ciccone } \\
\text { et al: } 178 \text { NETT: } 116\end{array}$ \\
\hline \multicolumn{5}{|c|}{$\mathrm{FEV}_{1}($ mean $\pm \mathrm{SD}(\mathrm{L}), \%$ predicted $)$} \\
\hline Ciccone et al & $0.7 \pm 0.3(26 \%)$ & $1.1 \pm 0.5(39 \%)$ & $1.0 \pm 0.5(38 \%)$ & $0.9 \pm 0.5(34 \%)$ \\
\hline NETT & $0.7 \pm 0.2(26 \%)$ & $1.0 \pm 0.4(37 \%)$ & $1.0 \pm 0.3(35 \%)$ & $0.9 \pm 0.3(33 \%)$ \\
\hline \multicolumn{5}{|c|}{$\mathrm{RV}($ mean $\pm \mathrm{SD}(\mathrm{L}), \%$ predicted $)$} \\
\hline Ciccone et al & $5.8 \pm 1.3(277 \%)$ & $4.0 \pm 1.2(189 \%)$ & $4.1 \pm 1.3(193 \%)$ & $4.2 \pm 1.3(198 \%)$ \\
\hline NETT & $5.0 \pm 1.0(226 \%)$ & $3.4 \pm 1.0(157 \%)$ & $3.6 \pm 1.0(163 \%)$ & $3.7 \pm 1.0(163 \%)$ \\
\hline \multicolumn{5}{|c|}{ Six-minute walk (feet, mean \pm SD) } \\
\hline Ciccone et al & $1142 \pm 291$ & $1345 \pm 316$ & $1341 \pm 310$ & $1271 \pm 305$ \\
\hline NETT & $1166 \pm 317$ & $1354 \pm 304$ & $1339 \pm 329$ & $*$ \\
\hline
\end{tabular}

Ciccone et al had $8 \%$ of heterogeneous non-upper lobe predominant cases. *Six-minute walk: data available up to 24 months. 

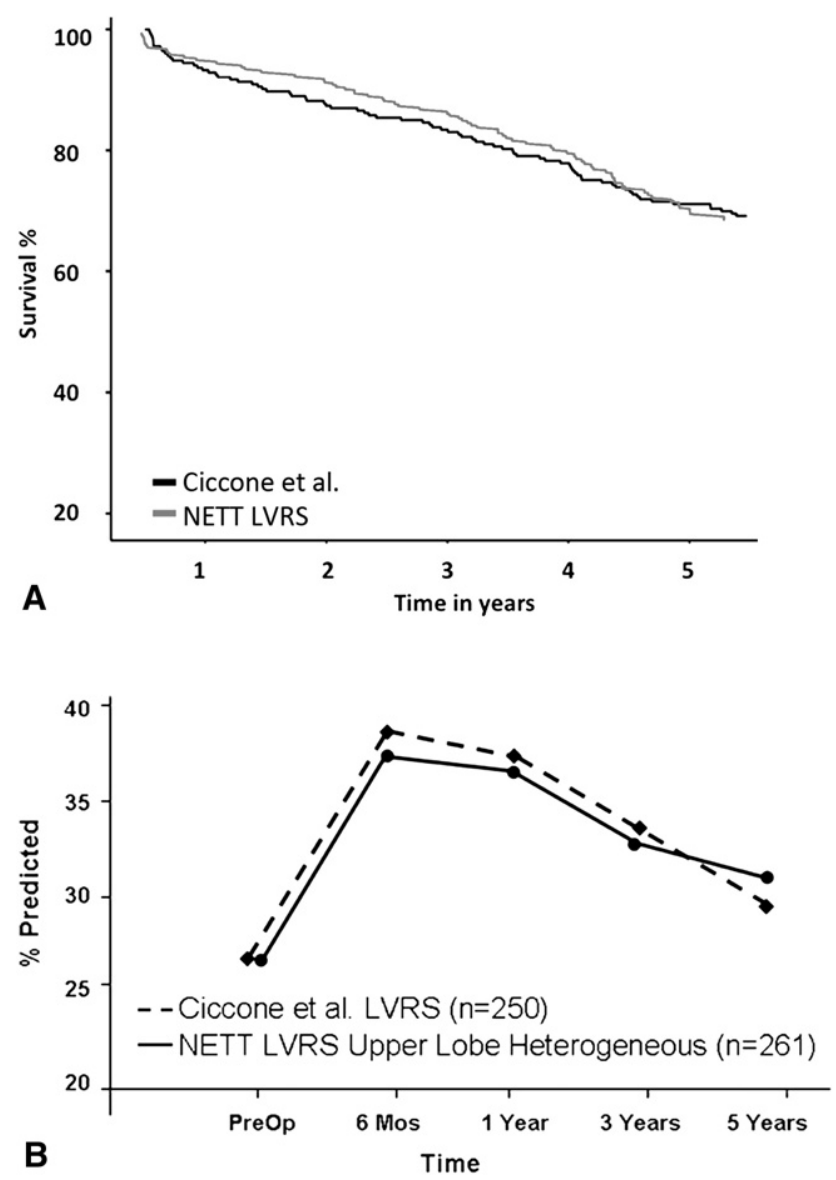

FIGURE 2. Results following bilateral LVRS, NETT heterogeneous upper lobe predominant versus Ciccone and associates. ${ }^{3}$ A, Kaplan Meier survival. $\mathrm{B}, \mathrm{FEV}_{1} \%$ predicted. NETT, National Emphysema Treatment Trial; $L V R S$, lung volume reduction surgery; $F E V_{1}$, forced expiratory volume in 1 second.

of the trial, misunderstanding of the initial interim report relating to patients at high risk of death after the procedure, and a complex statistical presentation of outcome results in a manner difficult to interpret and to compare with other reported series.

Release of the long-term follow-up data of the NETT trial provided the opportunity to analyze the data in a manner consistent with previous reports and to clarify and reanalyze data subsets in a manner that allowed more valid comparison with other series, and in keeping with the hypothesis of the NETT trial.

As previously noted, the NETT protocol defined 2 separate methods of defining the pattern and severity of emphysema in the lungs. The first was based on which part of the lung was most affected ("predominance") and the second, based on the 0 to 4 severity score, yielded a classification of either heterogeneous or homogenous. Thus some patients assigned to the upper lobe predominance group were classified as heterogeneous and others were classified as homoge- neous. This contributed to the confusion in interpreting the data presented in the NETT publications. In the initial publication, "Patients at High Risk of Death after Lung-VolumeReduction Surgery," patient subsets were classified only as having either a homogenous or a heterogeneous pattern of emphysema. ${ }^{11}$ Moreover, most of the patients reported in that publication were in fact ineligible under the original NETT selection criteria and were enrolled only after these criteria were significantly changed in the midst of the trial.

For the reporting of the NETT outcome as a whole ${ }^{12}$ patients were classified as having either upper lobe predominance or non-upper lobe predominance. Neither of the initial 2 NETT publications reported results on the subset of patients who met NETT hypothesis criteria.

Applying the NETT hypothesis to the NETT data reported to date reveals that only $46 \%$ of the 508 patients who received bilateral LVRS met the hypothesis selection criteria, and results in this group demonstrate better outcomes than any group reported by the NETT to date, in terms of lung function, exercise tolerance, quality of life, and survival. Furthermore, only this "target" group of patients, as identified in this article, can be considered to be a group prospectively identified before initiating the trial, as all other subgroups reported were retrospectively defined.

Our original and ongoing presumption as to the most appropriate candidates for bilateral LVRS has been that such candidates should have a heterogeneous pattern of emphysema to provide "target" areas that can be excised with as little loss of functioning lung as possible. Our report of the initial 250 consecutive patients undergoing bilateral LVRS highlights the fact that the vast majority $(92 \%)$ of patients with a heterogeneous pattern of emphysema who were deemed suitable for bilateral LVRS have upper lobe predominant disease.

The opportunity to compare the results of our previously reported consecutive case series with similar patients in a randomized trial having similar characteristics was extremely valuable. The 261 patients in the NETT group had a median follow-up of 4.3 years compared with a median of 4.4 years in the consecutive case series. The baseline $\mathrm{FEV}_{1}$ and 6-minute walk distance for the 2 groups were identical. The baseline RV for the case series $(5.8 \pm 1.3 \mathrm{~L})$ was somewhat higher than the NETT group $(5.0 \pm 1 \mathrm{~L})$, indicating perhaps more stringent selection criteria in the case series. As the NETT patients were by definition Medicare patients, the average age of the NETT group (66.5 years) was somewhat older than that of the case series (62 years). The results in the 2 series, however, are virtually identical with respect to survival curves, improvement in $\mathrm{FEV}_{1}$, and reduction in $\mathrm{RV}$. This comparison demonstrates that the multicenter NETT trial was able to achieve very similar results to those achieved in similar patients in a single-center study. It might be concluded, therefore, that the results of the multicenter, randomized NETT trial confirmed the results obtained with 
TABLE 3. Surgery versus medical therapy: Bilateral LVRS in homogeneous group

\begin{tabular}{|c|c|c|c|c|c|}
\hline & $\begin{array}{c}\text { Preop: } \\
\text { Surg }=257 \\
\text { MT }=256\end{array}$ & $\begin{array}{c}\text { Six months: } \\
\text { Surg = 211 } \\
\text { MT = 185 }\end{array}$ & $\begin{array}{c}\text { One year: } \\
\text { Surg }=170 \\
\text { MT }=153\end{array}$ & $\begin{array}{c}\text { Two years: } \\
\text { Surg }=142 \\
\text { MT }=118\end{array}$ & $\begin{array}{c}\text { Three years: } \\
\text { Surg }=82 \\
\text { MT }=67\end{array}$ \\
\hline \multicolumn{6}{|c|}{$\mathrm{FEV}_{1}($ mean $\pm \mathrm{SD}(\mathrm{L}), \%$ predicted $)$} \\
\hline Surg Homog & $0.8 \pm 0.2(27 \%)$ & $0.9 \pm 0.3(33 \%) \ddagger$ & $0.8 \pm 0.3(32 \%)$ & $0.8 \pm 0.2(30 \%)$ & $0.8 \pm 0.4(29 \%)$ \\
\hline MT Homog & $0.8 \pm 0.2(27 \%)$ & $0.8 \pm 0.2(27 \%)$ & $0.8 \pm 0.2(26 \%)$ & $0.8 \pm 0.3(27 \%)$ & $0.7 \pm 0.3(26 \%)$ \\
\hline \multicolumn{6}{|c|}{$\mathrm{RV}($ mean $\pm \mathrm{SD}(\mathrm{L}), \%$ predicted $)$} \\
\hline Surg Homog & $4.9 \pm 1.0(222 \%)$ & $3.8 \pm 1.0(172 \%) \ddagger$ & $3.9 \pm 1.1(176 \%) \ddagger$ & $4.0 \pm 1.1(179 \%) \ddagger$ & $4.2 \pm 1.3(187 \%) \dagger$ \\
\hline MT Homog & $5.0 \pm 1.3(226 \%)$ & $4.9 \pm 1.2(219 \%)$ & $4.9 \pm 1.3(217 \%)$ & $4.9 \pm 1.3(214 \%)$ & $4.8 \pm 1.1(210 \%)$ \\
\hline \multicolumn{6}{|c|}{$\mathrm{UCSD}($ mean $\pm \mathrm{SD})$} \\
\hline Surg Homog & $65 \pm 19$ & $49 \pm 23 \ddagger$ & $47 \pm 23 \ddagger$ & $52 \pm 23 \ddagger$ & $52 \pm 24 \ddagger$ \\
\hline MT Homog & $68 \pm 18$ & $67 \pm 20$ & $68 \pm 19$ & $68 \pm 20$ & $69 \pm 21$ \\
\hline \multicolumn{6}{|c|}{ St George (mean $\pm \mathrm{SD}$ ) } \\
\hline Surg Homog & $65 \pm 19 \ddagger$ & $49 \pm 23 \ddagger$ & $47 \pm 23 \ddagger$ & $52 \pm 23^{*}$ & $52 \pm 24$ \\
\hline MT Homog & $58 \pm 13$ & $56 \pm 14$ & $57 \pm 13$ & $57 \pm 14$ & $57 \pm 14$ \\
\hline \multicolumn{6}{|c|}{ Workload (watts, mean \pm SD) } \\
\hline Surg Homog & $35 \pm 21$ & $43 \pm 21$ & $42 \pm 22$ & $41 \pm 22$ & $38 \pm 24$ \\
\hline MT Homog & $35 \pm 21$ & $39 \pm 22$ & $38 \pm 23$ & $36 \pm 22$ & $36 \pm 21$ \\
\hline
\end{tabular}

Surg, Surgery; Homog, homogenous; $M T$, medical therapy; $F E V_{l}$, forced expiratory volume in 1 second; $S D$, standard deviation; $R V$, residual volume; $U C S D$, University of California San Diego, Shortness of Breath Questionnaire is a 24-item questionnaire about dyspnea. Total score ranges from 1 to 120 . Lower scores indicate less shortness of breath. The St George's Respiratory Questionnaire is a 51-item questionnaire on the health-related quality of life with regard to respiratory symptoms. Total score ranges from 0 to 100 . Lower score indicates better health-related quality of life. ${ }^{*} P \leq .05$ for paired comparison with MT scores at same follow-up. $\dagger P \leq .002$ for paired comparison with MT scores at same follow-up. $\ddagger P \leq .001$ for paired comparison with MT scores at same follow-up.

a nonrandomized consecutive case series. Furthermore, it might be concluded that the results obtained in the case series, in which follow-up data were far more complete than in the multicenter trial, confirmed the validity of the randomized trial despite considerable missing data. The most important additional value obtained from the randomized nature of the NETT trial is the marked benefit of bilateral LVRS in well-selected patients when compared with the medical control group consisting of similar patients.

A second benefit of this report is the opportunity to refine appropriate selection criteria for LVRS candidates. The NETT trial included 513 patients with homogeneous disease, of whom 257 received LVRS and 256 received medical management. For these patients, surgery was associated with a $10 \%$ operative mortality, offered no apparent longterm survival benefit, and resulted in less objective and subjective benefit than observed in the NETT patients with heterogeneous upper lobe predominant disease. However, further analysis and further experience with other subgroups of patients enrolled in the NETT trial might demonstrate benefit from bilateral LVRS or that the application of a unilateral procedure for some of these patients might be appropriate. Reports of highly selected patients with a homogeneous morphology and patients undergoing unilateral LVRS have certainly suggested potential benefit.

One of the most controversial aspects of the NETT trial was the manner in which the data were analyzed and reported, a method quite dissimilar from the methods usually used when analyzing the results of surgical procedures. The methodology used to describe statistical differences between the surgical and medical groups compared the probability of each group achieving a specified target at different periods of time. Data were presented with complex histograms, and the magnitude of benefit achieved by patients at each of the chosen time periods was difficult to describe in simple terms. This is illustrated with the NETT analysis of the maximum workload achieved, which was 1 of the 2 primary end points of the NETT trial. Workload, as determined by cycle ergometry, was measured in watts and statistical analysis between treatment groups determined whether or not one group or the other was more likely to achieve a 10-watt improvement over baseline. However, as noted in the NETT report, there are differences between men and women in baseline exercise capacity. Thus, to achieve the target of a 10-watt increase, women would have to show a $40 \%$ improvement whereas men only a $25 \%$ improvement. In the NETT report ${ }^{13}$ (Table 3), the 2 -year results in terms of improvement in exercise capacity shows that $21 \%$ of patients with upper lobe predominant disease achieved a 10-watt or higher increment in exercise tolerance whereas only $2 \%$ of the medical group achieved this magnitude of benefit. By comparison, our analysis (see Table 1) demonstrates that for the "target", LVRS group, the mean increase over baseline in maximum workload was $33 \%$ at 6 months, $36 \%$ at 1 year, and $36 \%$ at 2 years. We believe that this type of data presentation is more easily interpreted and more easily explained when describing the benefits of LVRS to physicians and patients.

Much of the controversy surrounding the previously published conclusions of the NETT trial is due, at least in part, to the fact that less than half of the patients enrolled in the trial met the eligibility criteria consistent with the NETT 
hypothesis as to who would likely benefit from LVRS, but there was no stratification or separate analysis of this prospectively identified group of patients. We hope that this report, using the valuable repository of data collected by the NETT trial, will help clarify the role of LVRS for selected patients with emphysema. This is particularly important inasmuch as none of the endoscopic procedures proposed in an attempt to achieve a "volume reduction" effect (valves, installation of adhesives, airway bypass) has demonstrated sufficient efficacy to warrant Food and Drug Administration approval at this time. Coupled with the reduction in priority status for patients with chronic obstructive pulmonary disease seeking a lung transplant, this potentially increases the importance of LVRS for the management of selected patients with severe chronic obstructive pulmonary disease.

\section{CONCLUSIONS}

The current analysis of data obtained by the NETT improves our ability to understand and clarify the current role of LVRS. This analysis confirms that the NETT hypothesis was indeed correct and that patients with heterogeneously distributed upper lobe predominant emphysema achieved significant benefit in terms of survival and functional improvement. We also conclude that for patients with a homogeneous pattern of emphysematous destruction, bilateral LVRS offers no survival advantage and produces much less functional improvement than in the heterogeneous upper lobe predominant group.

This analysis of the NETT results confirms, with remarkable concordance, the previous report by Ciccone and associates ${ }^{3}$ of a 250 consecutive case series of bilateral LVRS. We suspect that the similarity of results will contribute to the ongoing debate as to the relative merits of case-controlled series versus randomized clinical trials for surgical procedures.

\section{References}

1. Brantigan O, Mueller E, Kress M. A surgical approach to pulmonary emphysema. Am Rev Respir Dis. 1959;80:194-202.

2. Cooper JD, Trulock EP, Triantafillou AN, Patterson GA, Pohl MS, Deloney PA, et al. Bilateral pneumonectomy (volume reduction) for chronic obstructive pulmonary disease. J Thorac Cardiovasc Surg. 1995;109:106-19.

3. Ciccone AM, Meyers BF, Guthrie TJ, Davis GE, Yusen RD, Lefrak SS, et al. Long-term outcome of bilateral lung volume reduction in 250 consecutive patients with emphysema. J Thorac Cardiovasc Surg. 2003;125:513-25.

4. Bloch KE, Georgescu CL, Russi EW, Weder W. Gain and subsequent loss of lung function after lung volume reduction surgery in cases of severe emphysema with different morphologic patterns. J Thorac Cardiovasc Surg. 2002;123:845-54.

5. Criner GJ, Cordova FC, Furukawa S, Kuzma AM, Travaline JM, Leyenson V, et al. Prospective randomized trial comparing bilateral lung volume reduction surgery to pulmonary rehabilitation in severe chronic obstructive pulmonary disease. Am J Respir Crit Care Med. 1999;160:2018-27.

6. Sciurba FC, Rogers RM, Keenan RJ, Slivka WA, Gorcsan J 3rd, Ferson PF, et al. Improvement in pulmonary function and elastic recoil after lung-reduction surgery for diffuse emphysema. N Engl J Med. 1996;334:1095-9.

7. McKenna RJ Jr, Brenner M, Fischel RJ, Gelb AF. Should lung volume reduction surgery be unilateral or bilateral? J Thorac Cardiovasc Surg. 1996;112:1331-9.

8. Miller JI Jr, Lee RB, Mansour KA. Lung volume reduction surgery: lessons learned. Ann Thorac Surg. 1996;61:1464-9.
9. Daniel TM, Chan BK, Bhaskar V, Parekh JS, Walters PE, Reeder J, et al. Lung volume reduction surgery. Case selection, operative technique, and clinical results. Ann Surg. 1996;223:526-33.

10. The National Emphysema Treatment Trial Research Group. Rationale and design of The National Emphysema Treatment Trial: a prospective randomized trial of lung volume reduction surgery. Chest. 1999;116:1750-61.

11. The National Emphysema Treatment Trial Research Group. Patients at high risk of death after lung-volume-reduction surgery. N Engl J Med. 2001;345:1075-83.

12. Fishman A, Martinez F, Naunheim K, Piantadosi S, Wise R, Ries A, et al. A randomized trial comparing lung-volume-reduction surgery with medical therapy for severe emphysema. N Engl J Med. 2003;348:2059-73.

13. Naunheim KS, Wood DE, Mohsenifar Z, Sternberg AL, Criner GJ, DeCamp MM, et al. Long-term follow-up of patients receiving lung-volume-reduction surgery versus medical therapy for severe emphysema by the National Emphysema Treatment Trial Research Group. Ann Thorac Surg. 2006;82:431-43.

14. Minutes of NETT Steering Committee. March 24-25, 1997.

\section{Discussion}

Dr Rodney J. Landreneau (Pittsburgh, $P a$ ). This is a very nice rehashing of what we have known for many years about LVRS. I can remember when Jim Luketich joined me at the University of Pittsburgh back in 1995 from Memorial. He believed it was like a laminar air flow coming into the intensive care unit because of all the negative suction coming from the chest tubes in place for our LVRS patients. That was back in 1995 or so, right, Jim? By 1998, however, we had come to recognize that we were operating on a lot of people erroneously, and then the NETT trial began.

If you look at the work of Dr Cooper's group, who is leading us out of Washington University, Larry Kaiser's work at the University of Pennsylvania, Mike Mack in Dallas, our work at the University of Pittsburgh, Dan Miller at Louisville at that time, and Dr Weder in Europe, we all had come to this conclusion by 1998 that this is the group of people we should be operating on. Again, we were led by Dr Cooper's group, and I re-emphasize their article from 2002.

I think that what has occurred is really concerning, as I hope Dr Cooper will re-reiterate. The trend of publications off the NETT trial was unfortunate and has basically resulted in the death or near death of LVRS for a number of patients who could have benefited over the past 6 to 7 years. To me, it is the abomination of health care at its finest through government intervention, and I think that we need to guard against subsequent forays like this in the future. Although we certainly need to avoid unnecessary surgery and to economize our use of the health-care dollar, I think that this is just a case in point on how to kill an advantageous intervention for a select group of patients. I look forward to hearing Dr Cooper's comments.

Dr Cooper. Rod, thank you very much.

As I think you indicated, the point of this presentation is not to criticize the NETT trial. It is to try to extract from it finally, and for the first time ever, exactly what it set out to do. Who are the most appropriate patients? There was a reason for that hypothesis, Rod, and that was based on what you and I and others had done, and the hypothesis was based correctly on the experience to date. However, the hypothesis specifically said upper lobe predominant, with a heterogeneous pattern. The NETT trial developed 2 separate criteria for the patterns of emphysema. One defined homogeneous and heterogeneous and the other defined predominance -2 totally different criteria. But the hypothesis said that both have to be present: predominance, that is, the upper lobe should be worse than the rest of the zones, and also heterogeneous, as defined by the severity grade developed by the NETT trial. Each of the 3 lung zones was 
graded in terms of severity on a scale of 0 to 5 . For the upper lung zone to be defined as heterogeneous, it had to be at least 2 severity grades worse than the other zones on the same side. The problem was that when they reported the data, they contaminated the data, so that their second publication was only based on predominance, and many of the upper lobe predominant patients had homogeneous distribution by their definition, and they mixed homogeneous and heterogeneous patients together when reporting the data. It was only because there was a hypothesis and because they specifically outlined how to define upper lobe predominance and heterogeneity that we can present this information and not be accused of post hoc examination of the data. That was probably the most serious charge against the final NETT report, that they diced and sliced the data retrospectively. Therefore, I appreciate the comment. Yes, there may be other patients who require individual judgment, but the NETT trial showed in a randomized fashion that if the right patients are selected, the right result is achieved. And I think it is astonishing that a multicenter trial, comprising 17 centers, was able to accomplish exactly, identically the same results that we obtained with our original single-center report. That usually does not happen. Multicenter trials usually do not achieve the same results. They did. They did it beautifully. They achieved exactly the same results. The problem was that the manner in which it was presented was very, very confusing and confused the surgeons, the referring physicians, and the patients. I guess the message is that if the right patients are chosed, the right results will be achieved.

Dr Robert J. Cerfolio (Birmingham, Ala). I have a quick question for you, Joel. We all know what has happened. The pulmonologists have sort of pulled the plug on LVRS. I do 1 or 2 a year. The question now is with the new Spiration valves (Spiration, Inc, Redmond, Wash) or the other endobronchial valves. Is that going to increase the number of people coming from LVRS, and if it does, who do you think should get the valve, endobronchial therapy, and who should get LVRS, and why?

Dr Cooper. Well, isn't it interesting that the pulmonologists who pooh-poohed LVRS nonetheless got the message that if you reduce overinflation in emphysema patients, you transform their lives to the point that they got interested in interventional procedures. But, as you may or may not know, one valve company's trial was not approved. They are out of business. The second valve company's preliminary data showed no benefit, and I have little doubt that their pivotal trial will show no benefit. The third company, which developed the gluing technique, I believe is no longer operational. The only thing that is still standing, and here I have a conflict, is the airway bypass, which was really not designed for patients with heterogeneous distribution but was designed for those patients with homogeneous distribution. Ideally, the fact that there does not appear to be a bronchoscopically effective alternative and the fact that the NETT trial shows that if you focus on the right patients, you get excellent results, will rekindle interest in LVRS.

I would also like to note that the NETT report retrospectively broke up the upper lobe predominant patients into 2 subgroups based on the preoperative exercise capacity defined as either high or low exercise capacity. When you now look at the appropriate upper lobe predominant patients, namely, those who meet the criteria of upper lobe predominant and heterogeneous, then the outcome after LVRS is exactly the same whether or not the patient had a high or low exercise capacity. The message is that the most appropriate patients for LVRS are those who have an upper lobe predominant, heterogeneous distribution pattern of emphysema. I believe that it is necessary to promulgate the NETT data in a transparent fashion and to eliminate what I like to call the toxic data included in their analysis, namely, the inclusion of both heterogeneous and homogeneous distribution in what was called their upper lobe predominant patients. This will highlight the true value of the NETT study and lead to a resurgence of appropriate use of LVRS.

Dr Cerfolio. That was a circuitous answer. I think the valves do work. So my question is, in what patient who comes to you would you put the endobronchial valve in and to whom would you recommend LVRS and why?

Dr Cooper. You are right. First of all, the question is, will they be allowed to sell any valves? One company is out. Maybe they will. I have often said to a patient, "If I could only wish away your upper lobes, I know you would be better. You would have less hyperinflation. Your work at breathing would be better. I can't wish it away. I have to subject you to an operation. You've got cardiac issues. You've got vascular issues. I don't think it's worth the risk in your case." That is the patient, in my opinion, who might be considered for the valves if they are approved. That is the group of patients for whom I would like to have an alternative to LVRS.

Dr Walter Weder (Zurich, Switzerland). You write in your abstract that a lot of data are missing in the medical group of the NETT. Could you elucidate this?

Dr Cooper. I had 3 slides. Flip through those slides.

Dr Weder. Were these data relevant or irrelevant?

Dr Cooper. Dr Weder, I was very surprised to see how much data were missing, so much so that we were unable to carry out our analysis after the third year. It turns out that although the NETT trial was 5 years, they stopped collecting data (and maybe that was built into it) after 2 to 3 years. But, Walter, there were 2 primary end points. One is survival. Of course they had death records. That's immaculate. They clearly have $100 \%$ data there. The other was workload. This slide says pulmonary function tests and functional data. Functional data was the maximum workload. As you can see, functional data are missing on $30 \%$ of the patients who are alive at least 1 year after randomization, and by 3 years more than $35 \%$ of patients are missing functional data. When I showed this to a statistician, he pointed out that not only does the NETT trial validate our previously reported data, but in fact our data validate the NETT trial data because we had less than $10 \%$ missing data at every time point to 5 years, and since we had the same apparent results as the NETT trial, it means that the missing data in the NETT trial were probably not critical. Specifically, it probably means that the missing data did not selectively represent patients with a bad result; therefore, they did not necessarily bias the outcome. Nonetheless, it was a surprise to find out how much missing data there was in this highly funded, well-organized trial.

Dr David M. Follette (Sacramento, Calif). Joel, I want to compliment you, and I would like to have you make a comment on another point. One of the great contributions I found from your work is that it has greatly expanded my ability to operate on patients with lung cancer. As you well know, there is a subgroup of patients with cancer who are said to be inoperable because of poor pulmonary 
function. Because of your work, which was nicely summarized today, I have used similar criteria to evaluate these cancer patients. In those patients with heterogeneous disease with isolated lung cancers in the poorly perfused areas, I have performed resection of the area. These patients have not only had great results with respect to their cancer but have also had remarkable improvement in their pulmonary function and exercise capacity. Dr Cooper, have you looked at this subgroup of patients with a diagnosis of severe emphysema and lung cancer?

Dr Cooper. We have. We actually had an initial report of our first 20 patients- $-\mathrm{FEV}_{1} \mathrm{~s}$ less than $30 \%$, all oxygen-dependentwho underwent an anatomic lobectomy with or without additional resections for volume reduction purposes. I think there was 1 death and an excellent survival curve.

I believe we have obtained 3 benefits from volume reduction surgery other than the benefit for the LVRS patients themselves: (1) improved anesthetic and pain management with high-risk patients undergoing lung cancer, (2) the benefit of exercise rehabilitation in patients with emphysema, and (3) the recognition that if the cancer is in the right place, a simple lobectomy or lobectomy plus some other volume reduction not only gets rid of the cancer but also gives significant improvement in lung function. In our series there was an average of $38 \%$ improvement in the first second vital capacity in the lobectomy patients whose preoperative $\mathrm{FEV}_{1}$ was less than $30 \%$, who were oxygen-dependent, and who were not candidates for lobectomy for their cancer, based solely on their risk factors.

One of the old-time surgeons told me about a patient who had demanded a lobectomy for his cancer. It was an upper lobe cancer, and the patient had severe emphysema and was a very high risk. The surgeon said, "I went ahead and did it, and I got rid of the cancer and he breathed much better." If he had told us that years ago, we would have started LVRS earlier. 